\title{
On Regularity and Flatness
}

\section{Nazar H. Shuker}

nazarh_2013@yahoo.com

Dept. of Mathematics

\section{College of Computer Sciences and Mathematics}

University of Mosul, Iraq

Received on: 19/2/2002

Accepted on: 02/06/2002

\section{ABSTRACT}

A ring $\mathrm{R}$ is called a right SF-ring if all its simple right $\mathrm{R}$ modules are flat. It is well known that Von Neumann regular rings are right and left SF-rings. In this paper we study conditions under which SF-rings are strongly regular. Finally, some new characterstic properties of right SF-rings are given.

Keywords: modules, flat, Von Neumann regular rings.

$$
\text { حول الانتظام والتسطح }
$$

كلية علوم الحاسوب والرياضيات، جامعة الموصل

تاريخ القبول: 2002/06/02

تاريخ الاستلام: 2002/02/19

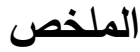

يقال للحلقة R بأنها من النمط SF اليمنى ، إذا كان كل مقاس بسيط ايمن

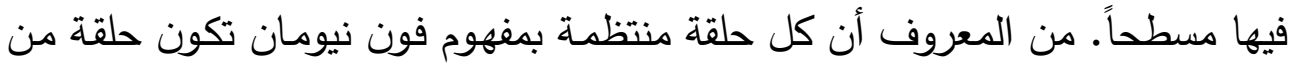

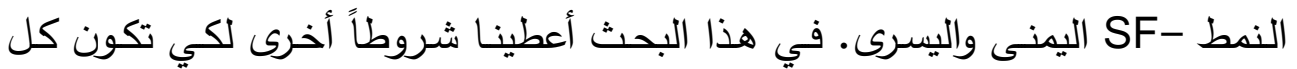
حلقة من النمط SF- اليمنى حلقة منتظمة بقوة ـ ومن النتائج الأخرى التي حصلنا عليها هي خواص أخرى جديدة للحلقات من النمط المط -SF اليمنى. الكلمات المفتاحية: المقاسات، المسطحة، حلقة منتظمة بمفهوم فون نيومان. 


\section{INTRODUCTION}

In this paper all rings are assumed to be associative with identity, and all modules are unital right R-modules.

Following [2], a ring $\mathrm{R}$ is called a right (left) SF-ring if all of its simple right (left) R-modules are flat . It is well known that a ring $\mathrm{R}$ is Von Neumann regular if and only if every right (left) $\mathrm{R}$-module is flat [3]. Ramamurthi in [8] asked whether left and right SF-ring is Von Neumann regular. Many authors have given various conditions for SF-rings to be regular (see, e.g. Chen [1], Ming [4], Rege [9] and $\mathrm{Xu}$-[10] ). In this paper, to the list of equivalent conditions, we shall add several news. We recall that:

1- $\quad A$ ring $R$ is called reduced if $R$ contains no non-zero nilpotent elements.

$2-\mathrm{R}$ is said to be Von Neumann regular (or just regular) if $a \in a \mathrm{R} a$ for every $a \in \mathrm{R}$, and $\mathrm{R}$ is called strongly regular if $a \in a^{2} \mathrm{R}$. Clearly, every strongly regular ring is a regular reduced ring.

3- $\mathrm{R}$ is said to be right duo-ring if every right ideal is a two-sided ideal.

4-r(a) and L(a) will denote right and left annihilator of $a$ respectively.

5-Following [9], for any ideal I of R, R/I is flat if and only if for each $a \in \mathrm{I}$, there exists $\mathrm{b} \in \mathrm{I}$ such that $a=\mathrm{b} a$.

6-Y and $\mathrm{J}$ will stand for the right singular ideal and Jacobson radical of $R$.

\section{RINGS WHOSE SIMPLE MODULES ARE FLAT}

Following [7], a ring $\mathrm{R}$ is called ERT-ring if every essential right ideal of $\mathrm{R}$ is a two-sided ideal.

Ming [6] proved the following:

Proposition 2.1. If $R$ is a right duo-ring then $R / Y$ is a reduced ring.

We use a similar method of proof in Prop.2.1 to establish the following lemma. 
Lemma 2.2: If $R$ is an ERT-ring, then $R / Y$ is a reduced ring.

Proof. Suppose that $\mathrm{R} / \mathrm{Y}$ is not reduced, then there exists an element

$\mathrm{Y} \neq a+\mathrm{Y} \in \mathrm{R} / \mathrm{Y}, a \in \mathrm{R}$, such that $(a+\mathrm{Y})^{2}=\mathrm{Y}$. This implies that $a \notin \mathrm{Y}$ and $a^{2} \in \mathrm{Y}$. So $\mathrm{r}\left(a^{2}\right)$ is essential right ideal of $\mathrm{R}$. Since $\mathrm{R}$ is ERT, then $\mathrm{r}\left(a^{2}\right)$ is a two-sided ideal . Let I be any subideal of $\mathrm{r}\left(a^{2}\right)$

Such that

Iis/essential in $(a) \mathrm{I}$, this means that $\mathrm{r} \cap(a) \mathrm{r} \subseteq \mathrm{I} a$, then $(a) \mathrm{r} \subseteq$ $\mathrm{r}\left(a^{2}\right)$ and hence in $\mathrm{R}$, this contradicts $a \notin \mathrm{Y}$.

The following theorem gives the condition of being right SF-rings are strongly regular.

Theorem 2.3: Let $\mathrm{R}$ be a ring. Then the following are equivalent.

(1) R is strongly regular.

(2) $\mathrm{R}$ is a right SF- and ERT ring.

Proof. (1) $\Rightarrow(2)$ is obvious.

$\Rightarrow(1)$ By Lemma 2.2, R/Y is a reduced ring. We claim that $Y=0$. Suppose that $Y \neq 0$ then by [5], there exists $0 \neq y \in Y$ such that $\mathrm{y}^{2}=0$.

Let $\mathrm{M}$ be a maximal right ideal containing $\mathrm{r}(\mathrm{y})$. Since $\mathrm{r}(\mathrm{y})$ is an essential two-sided ideal of $\mathrm{R}$, then $\mathrm{M}$ must be an essential two-sided ideal of $\mathrm{R}$. On the other hand, since $\mathrm{R} / \mathrm{M}$ is flat module, and since $y \in M$, there exists $c \in M$ such that $y=y c$, whence $1-c \in r(y) \subseteq M$, yielding $1 \in M$ which contradicts $M \neq R$. This proves that $\mathrm{R}$ is a reduced ring. In order to show that $\mathrm{R}$ is regular we need to prove that $a \mathrm{R}+\mathrm{r}(a)=\mathrm{R}$ for any $a \in \mathrm{R}$. Suppose that $a \mathrm{R}+\mathrm{r}(a) \neq \mathrm{R}$, then there exists a maximal right ideal L containing $a \mathrm{R}+\mathrm{r}(a)$. But $a \in \mathrm{L}$ and $\mathrm{R} / \mathrm{M}$ is flat, there exists $\mathrm{b} \in \mathrm{L}$ such that $a=\mathrm{b} a$, whence $1-\mathrm{b} \in \mathrm{L}(a)=\mathrm{r}(a) \subseteq \mathrm{M}$. Yielding $1 \in \mathrm{M}$ which contradicts $\mathrm{L} \neq \mathrm{R}$. In particular $a \mathrm{r}+\mathrm{d}=1$, 
for some $\mathrm{r} \in \mathrm{R}$ and $\mathrm{d} \in \mathrm{r}(a)$, whence $a^{2} \mathrm{r}=a$. This proves that $\mathrm{R}$ is a strongly regular ring.

We now consider an other condition for right SF-ring to be strongly regular.

Theorem 2.4: Let $\mathrm{R}$ be a right SF-ring with every nilpotent element of $\mathrm{R}$ is central. Then $\mathrm{R}$ is strongly regular.

Proof. Let $a$ be a non-zero element in R with $a^{2}=0$, and let $\mathrm{M}$ be a maximal right ideal containing $\mathrm{r}(a)$. Since $a \in \mathrm{r}(a) \subseteq \mathrm{M}$, and since $\mathrm{R} / \mathrm{M}$ is flat, there exists $\mathrm{b} \in \mathrm{M}$ such that $a=\mathrm{b} a$. This implies that $1-\mathrm{b} \in \mathrm{L}(a)$. But every nilpotent is central gives $\mathrm{r}(a)=\mathrm{L}(a)$. Whence $1-\mathrm{b} \in \mathrm{r}(a) \subseteq \mathrm{M}$, yielding $1 \in \mathrm{M}$, and this contradicts $\mathrm{M} \neq \mathrm{R}$. Therefore, $\mathrm{R}$ is a reduced ring. By a similar method of proof used in Theorem 2.3, $\mathrm{R}$ is strongly regular.

\section{BASIC PROPERTIES}

Recall that a ring $\mathrm{R}$ is a right uniform if every right ideal of $\mathrm{R}$ is essential .

We are now in a position to give new characteristic properties of a right SF-ring.

Theorem 3.1: If $\mathrm{R}$ is a right SF- ring, then

1 - If $\mathrm{L}(a)=0$, then $a$ is a right invertable .

2 - Every reduced ideal of $\mathrm{R}$ is strongly regular.

3 - If $\mathrm{J}$ is reduced, then $\mathrm{J}=0$.

4- If $\mathrm{R}$ is a right uniform ring, then $\mathrm{R}$ is a division ring.

\section{Proof.}

(1) Let $a \in \mathrm{R}$ with $\mathrm{L}(a)=0$. If $a \mathrm{R} \neq \mathrm{R}$, there exists a maximal right ideal M containing $a \mathrm{R}$. Since $a \in \mathrm{M}$ and $\mathrm{R} / \mathrm{M}$ is flat, there exists $\mathrm{b} \in \mathrm{M}$, such that $a=\mathrm{b} a$. Whence $1-\mathrm{b} \in \mathrm{L}(a)=0$, yielding $\mathrm{L} \in \mathrm{M}$, which contradicts $\mathrm{M} \neq \mathrm{R}$. Therefore $a \mathrm{R}=\mathrm{R}$.

(2) Follows from Theorem 2.3. 
(3) Let $a \in \mathrm{J}$, then by (2) $\mathrm{J}$ is strongly regular, and hence there exists $\mathrm{b} \in \mathrm{J}$ such that $a=a^{2} \mathrm{~b}$. But $\mathrm{a} \in \mathrm{J}$ gives $(1-a \mathrm{~b}) \mathrm{u}=1$ for some $\mathrm{u} \in \mathrm{R}$, this implies that $\left(a-a^{2} \mathrm{~b}\right) \mathrm{u}=a$. Thus $a=0$, consequently, $\mathrm{J}=0$. (4) Suppose that $Y \neq 0$, then there exists a maximal right ideal $M$ containing $Y$. For any $0 \neq y \in Y$, gives $y \in M$, but $R / M$ is flat, then there exists $x \in M$ such that $y=x y$, whence $y \in r(1-x)$. On the other hand, since $\mathrm{R}$ is a right uniform, then $\mathrm{r}(1-\mathrm{x})$ is an essential right ideal of $R$. Thus $1-x \in Y \subseteq M$, this implies that $1 \in M$, contradicting $\mathrm{M} \neq \mathrm{R}$. Therefore, $\mathrm{Y}=0$. On the other hand, since $\mathrm{R}$ is uniform, then for every $a \in \mathrm{R}, \mathrm{r}(a)=0$, then by (1), $\mathrm{R}$ is a division ring. result.

Before closing this section, we present the following

Proposition 3.2: Let $\mathrm{R}$ be a reduced right SF- ring, for any $a$, $\mathrm{b} \in \mathrm{R}$ with $a \cdot \mathrm{b}=0$, then $\mathrm{r}(a)+\mathrm{r}(\mathrm{b})=\mathrm{R}$.

Proof. Suppose that $a \cdot \mathrm{b}=0$ and $\mathrm{r}(a)+\mathrm{r}(\mathrm{b}) \neq \mathrm{R}$. Then there exists a maximal right ideal $\mathrm{M}$ containing $\mathrm{r}(a)+\mathrm{r}(\mathrm{b})$. Since $a \in \mathrm{r}(\mathrm{b}) \subseteq \mathrm{M}$, and since $\mathrm{R} / \mathrm{M}$ is flat, there exists $\mathrm{c} \in \mathrm{M}$ such that $a$ $=\mathrm{c} a$, whence $1-\mathrm{c} \in \mathrm{L}(a)=\mathrm{r}(a) \subseteq \mathrm{M}$, yielding $\mathrm{L} \in \mathrm{M}$, which contradicts $\mathrm{M} \neq \mathrm{R}$.

Therefore $\mathrm{r}(a)+\mathrm{r}(\mathrm{b})=\mathrm{R}$. 


\section{REFERENCES}

[1] J. Chen (1991), On Von Neumann regular ring and SFrings , Math. Japon 36, pp. 1123-1127.

[2] Y.Hirano (1994), On rings whose simple modules are flat , Canad Math . Bull ., 37 (3), pp. 361-364.

[3] J.Lambek (1966), Lectures on rings and modules , Blaisdell, waltham, Massachusetts.

[4] R.Y.C.Ming (1980), On V-rings and prime rings , J.Algebra, 62 , pp. 13-20.

[5] R.Y.C . Ming (1983), On quasi-injectivity and Von Neumann regularity, Monatshefle . für Math . 95 , pp. 2532.

[6] R.Y.C.Ming (1987), On injectivity and p-injectivity , J Math kyoto univ. 27 (3) , pp. 439-452.

[7] R.Y.C.Ming (1998), A note on regular rings III. Riv. Math. Univ. Parma, 6(1), pp. 71-80.

[8] V.S. Ramamurthi (1975), On injectivity and flatness of certain cyclic modules, proc . Amer. Math. Soc., 48, pp. 21-25.

[9] M.B. Rege (1986), On Von Neumann regular rings and SF-rings, Math. Japonica, 31 (6), pp. 927-936.

[10] J.Xu (1991), Flatness and injectivity of simple modules over commutative ring, Comm . Algebra , 19 , pp. 535537. 